\title{
Probing the Unique Radiation Damage Response of Oxide Interfaces Using Multi-modal STEM Imaging, Diffraction, and Spectroscopy
}

Steven Spurgeon ${ }^{1}$, Bethany Matthews ${ }^{1}$, Tiffany Kaspar ${ }^{1}$, Weilin Jiang ${ }^{1}$, Jonathan Gigax ${ }^{2}$, Lin Shao $^{2}$, Vaithiyalingam Shutthanandan ${ }^{1}$ and Michel Sassi ${ }^{1}$

${ }^{1}$ Pacific Northwest National Laboratory, Richland, Washington, United States, ${ }^{2}$ Texas A\&M University, College Station, Texas, United States

Complex oxide materials are of great technological importance, underpinning the functionality of electronics and energy systems, as well as potential nuclear waste forms. When these materials are used in the highradiation environments of deep space and nuclear reactors, they are subject to lattice disordering and point defect formation processes that can significantly degrade their performance. While the radiation response of bulk oxides, such as the pyrochlores, has been extensively studied, far less attention has been paid to oxide interfaces, which possess distinct structural and chemical environments. In particular, cross-correlative methods to examine radiation-induced defect formation at the highest spatial and chemical resolution are needed to refine and bound theory models for damage accommodation. Here we describe an approach that harnesses the full array of scanning transmission electron microscopy (STEM) imaging, diffraction, and spectroscopy techniques to probe the onset of disorder in oxide interfaces at the atomic-to-nanoscale. We couple experiments with ab initio calculations, which identify the salient factors governing the radiation response of these materials and illuminate ways to tune defect formation pathways.

As shown in Figure 1, we have examined well-controlled, model interfaces between the archetypal perovskite oxide $\mathrm{SrTiO}_{3}$ (STO) and perovskite-like layered $\mathrm{La}_{2} \mathrm{Ti}_{2} \mathrm{O}_{7}$ (LTO) compounds grown using molecular beam epitaxy [1]. The LTO surface was exposed to controlled irradiation using $1 \mathrm{MeV} \mathrm{Zr+} \mathrm{ions} \mathrm{to} \mathrm{1,} \mathrm{2,} \mathrm{and} 4 \mathrm{dpa}$ dose and cross-sectional samples were extracted at each step using focused ion beam (FIB) preparation (Figures 1.A-D, respectively). Using a combination of STEM high-angle annular dark field (HAADF) and position-averaged convergent beam electron diffraction (PACBED), we tracked the evolution of structural disorder in this system, which begins at the bulk of both film and substrate and progresses toward the interface. We find that at the highest dose a pronounced crystalline band is retained at the substrate side of the interface, as indicated by the PACBED pattern. To explore the character of this region, we performed atomic-scale electron energy loss spectroscopy (EELS) of the $\mathrm{O} \mathrm{K}$ and $\mathrm{Ti} \mathrm{L}_{2,3}$ edges, which encode signatures of the local defect environment. As shown in Figure 2, we find substantial changes in the Ti crystal field splitting and a damping of the $\mathrm{O}$ edge features, which are consistent with the formation of large populations of oxygen vacancies. Finally, using the measured interface structure, we performed ab initio calculations for the energy of oxygen vacancy formation, which show a pronounced asymmetry toward the film rather than substrate side. Taken together, our results show how state-of-the-art imaging and spectroscopy can elucidate defect formation pathways in oxide interfaces in high-radiation and extreme environments. 

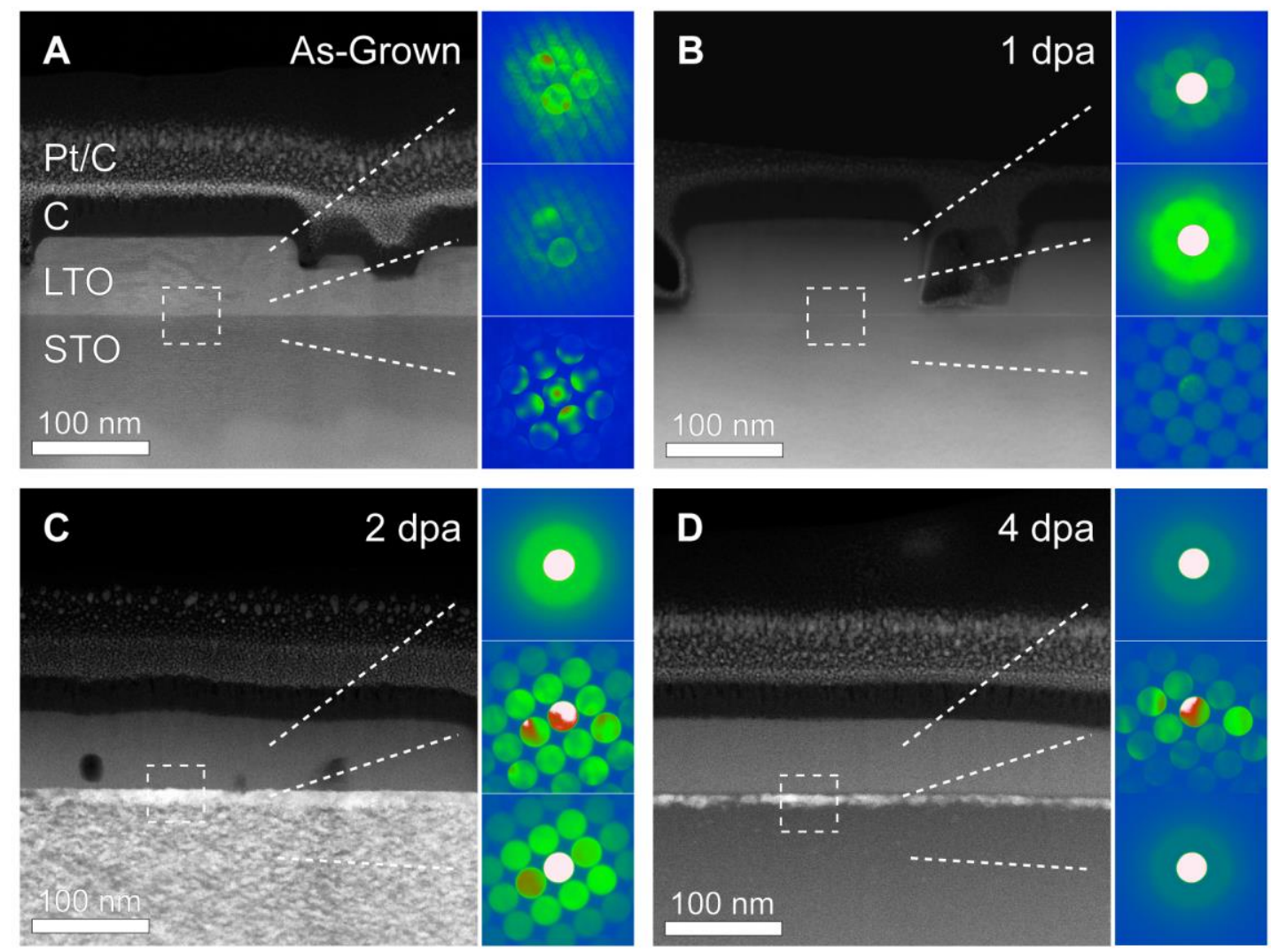

Figure 1. (A-D) Sequence of cross-sectional STEM-HAADF images and PACBED patterns taken from different parts of the sample as a function of dose spanning $0,1,2$, and $4 \mathrm{dpa}$, respectively.
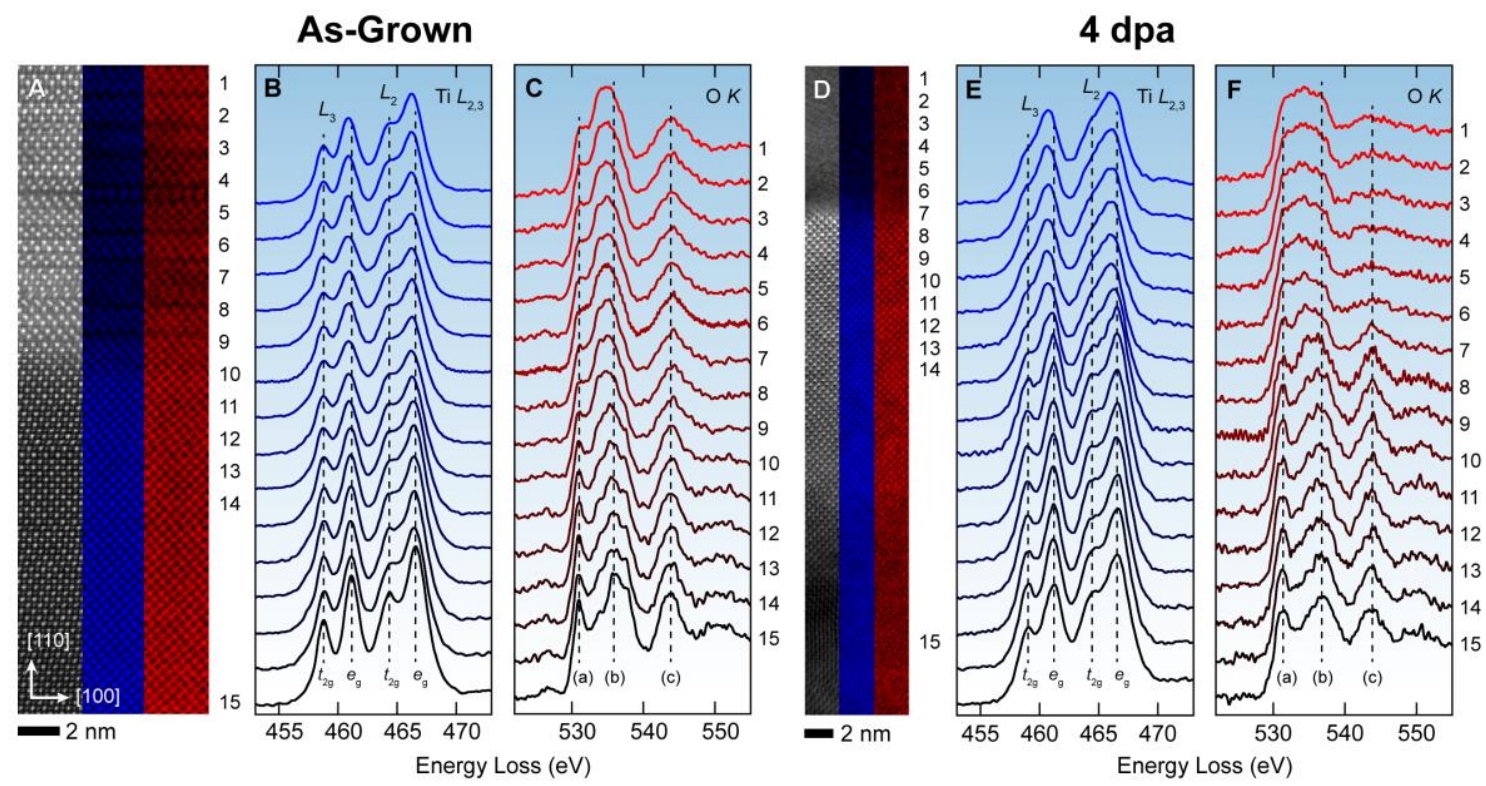

Figure 2. Atomic-scale EELS of the Ti L2,3 and O K edges for the as-grown (A-C) and 4 dpa (D-F) LTO / STO interfaces.

\section{References}

[1] Spurgeon, S. R. et al. Advanced Materials Interfaces (2020). DOI:10.1002/admi.201901944 